004 DIAGNOSTIC PERFORMANCE OF MYOCARDIAL BLOOD FLOW QUANTIFICATION IN CORONARY ARTERY DISEASE BY MAGNETIC RESONANCE

1,2Giorgos Papanastasiou, ${ }^{2}$ Michelle C Williams, ${ }^{2}$ Mark R Dweck, ${ }^{2}$ Shirjel Alam, ${ }^{1}$ Annette Cooper, 'Saeed Mirsadraee, ${ }^{1,2}$ David E Newby, ${ }^{1,2}$ Scott I Semple. ${ }^{1}$ Clinical Research Imaging Centre, University of Edinburgh, Edinburgh, UK; ${ }^{2}$ Centre for Cardiovascular Science, University of Edinburgh, Edinburgh, UK

\subsection{6/heartjnl-2016-309680.4}

Background Mathematical modelling of magnetic resonance (MR) perfusion imaging data allows myocardial blood flow (MBF) quantification and can potentially improve the diagnosis and prognostication of obstructive coronary artery disease (CAD). The diagnostic performance of distributed parameter (DP) modelling in detecting obstructive CAD has not yet been assessed. A model assessment in per vessel against per patient analysis has not been fully assessed yet in a single MR study. This work compares the diagnostic performance of DP modelling against the standard Fermi modelling, for the detection of obstructive CAD, in per vessel against per patient analysis.

Methods After informed consent, a pilot cohort of 28 subjects with known or suspected CAD underwent adenosine stress-rest magnetic resonance perfusion imaging at 3T. Data were analysed using Fermi and DP modelling against invasive coronary angiography and fractional flow reserve, acquired in all subjects. Obstructive CAD was defined as luminal stenosis of $\geq 70 \%$ alone, or luminal stenosis $\geq 50 \%$ and fractional flow reserve $\leq 0.80$.

Results On ROC analysis, the diagnostic performance of all methods was improved in per patient analysis. DP modelling outperformed the standard Fermi model, in per vessel and per patient analysis. In per patient analysis, DP modelling-derived MBF at stress demonstrated the highest sensitivity and specificity $(0.96,0.92)$ in detecting obstructive CAD, against Fermi modelling $(0.78,0.88)$ and visual assessments $(0.79,0.88)$, respectively. Conclusions DP modelling consistently outperformed Fermi modelling and showed that it may have merit for robustly stratifying patients with at least one vessel with obstructive CAD.

\section{NON-INVASIVE STRESS IMAGING AS A GATEKEEPER TO COMPLETE REVASCULARISATION IN STEMI PATIENTS WITH MODERATE BYSTANDER DISEASE AT PRIMARY PERCUTANEOUS CORONARY INTERVENTION}

\footnotetext{
1,2 Amardeep Ghosh Dastidar, ${ }^{1}$ Angus K Nightingale, ${ }^{1,2}$ Thomas W Johnson, ${ }^{1,3}$ Jonathan $\mathrm{CL}$ Rodrigues, ${ }^{1}$ Alexander Carpenter, ${ }^{1}$ Marco Cengarle, ${ }^{1}$ Anna Baritussio, ${ }^{1}$ Elisa McAlindon, ${ }^{1}$ Daniel X Augustine, ${ }^{1,2}$ Gianni Angelini, Julian Strange, ${ }^{1,2}$ Andreas Baumbach, ${ }^{1,2}$ Chiara Bucciarelli-Ducci. 'NIHR Bristol Cardiovascular Biomedical Research Unit, Bristol Heart Institute, UK; ${ }^{2}$ School of Clinical Sciences, St Michael's Hill, University of Bristol; ${ }^{3}$ School of Physiology and Pharmacology, Medical Sciences Building, University Walk, University of Bristol
}

\subsection{6/heartjnl-2016-309680.5}

Introduction $40 \%$ of patients presenting with STEMI have multivessel disease (MVD). Current international guidelines recommend revascularisation of the culprit artery only. However recent trials (PRAMI/CVLPRIT) have shown a superiority of complete in-hospital revascularisation. Objective: Assess the role of non-invasive stress imaging as a gatekeeper to complete revascularisation in STEMI patients with moderate bystander disease. Methods A registry study of consecutive patients who underwent Primary Percutaneous Coronary Intervention (P-PCI) of the culprit artery. Significant MVD was defined as non-culprit stenosis $\geq 50 \%$ in large proximal epicardial vessel, or $\geq 75 \%$ elsewhere (moderate if $50-74 \%$ stenosis in large proximal epicardial vessel, or $75-94 \%$ elsewhere). Non-invasive stress imaging was performed at 4 weeks. Patients with severe or critical bystander disease were excluded from the study. A simple cost analysis model was built for UK and USA.

Results 1,167 patients were included (74\% males, 64 years), $33 \%$ demonstrating MVD. $40 \%$ of MVD underwent stress CMR, and $36 \%$ a stress echocardiogram. The remaining 93 patients underwent direct revascularisation or were lost to follow up. Only $47 \%$ had evidence of inducible myocardial ischaemia in moderate bystander coronary artery disease (61/157 in stress CMR and 78/141 in stress echocardiogram). When PCI in MVD is performed only in the presence of inducible myocardial ischemia, there is saving. (UK or US).

Conclusions $<50 \%$ patients with moderate MVD at PPCI have inducible myocardial ischemia in the moderate bystander disease. Non-invasive stress imaging as a gatekeeper to complete revascularisation, may potentially reduce the number of PCIs, also resulting in a cheaper management strategy.

\section{MYOCARDIAL INFARCTION AND VIABILITY ASSESSMENT BY 12 LEAD ECG VS GOLD STANDARD CARDIAC MAGNETIC RESONANCE}

Amardeep Ghosh Dastidar, Alexander Carpenter, Jonathan Rodrigues, Alberto Palazzuoli, Catherine Wilson, Samantha Kestenbaum, Anna Baritussio, Andreas Baumbach, Angus Nightingale, Chiara Bucciarelli-Ducci. NIHR Bristol Cardiovascular Biomedical Research Unit, Bristol Heart Institute, Bristol, UK

\subsection{6/heartjnl-2016-309680.6}

Introduction Q-waves on 12 lead ECG is considered a marker of transmural myocardial infarction (MI) and is used universally. Late gadolinium enhancement (LGE) cardiovascular magnetic resonance (CMR) accurately identifies $\mathrm{MI}$ and has become the gold standard for the assessment of myocardial viability. Aim: Determine the diagnostic accuracy of Q-waves to identify MI and predict regionality. Ascertain the $\mathrm{CMR}$ predictors of $\mathrm{Q}-$ wave.

Methods Data collected on 498 consecutive patients (mean age 64 years, 71\% males) referred for CMR with suspected IHD. Patients with non-IHD were excluded. $Q$ waves in $\geq 2$ precordial leads from V1-V4 reflected LAD territory. Transmural infarction was defined as $>50 \%$ LGE.

Results 290 patients demonstrated MI, 157 transmural and 133 sub-endocardial based on LGE. Diagnostic accuracy of Q-wave as a marker of transmural MI was $66 \%$ and as a predictor of previous MI was 55\%. 126 had pathological Q-waves, 40\% in LAD territory, 55\% non-LAD and 5\% a combination. Of those with anterior (LAD) Q waves, 68\% demonstrated LAD territory LGE and in non-LAD Q waves, 67\% demonstrated a non-LAD territory infarct by LGE. On multivariate analysis, total scar score and $>75 \%$ thickness LGE were significant predictors of Q wave.

Conclusion Presence of pathological ECG Q-waves is not only a poor marker of myocardial scarring, but also a poor predictor of viability and regionality when compared to CMR. Presence of $\mathrm{Q}$ wave correlates only with total scar score and $>75 \%$ LGE. In clinical decision-making, clinicians need to be aware of the limitations of ECG Q-waves. 